\title{
On stability of rotation of the mean magnetic field of the Sun
}

\author{
V. I. Haneychuk, V. A. Kotov, and T. T. Tsap
}

\author{
Crimean Astrophysical Observatory, Nauchny, Crimea 98409, Ukraine \\ Isaak Newton Institute of Chile, the Crimean Branch \\ e-mail: han@crao.crimea.ua, vkotov@crao.crimea.ua
}

Received 30 September 2002 / Accepted 14 February 2003

\begin{abstract}
New data on the mean magnetic field of the Sun (MMFS) as a star measured at the Crimean Astrophysical Observatory in 1998-2001 are presented. The 34-year time series of the MMFS using similar data from three other observatories (1968-2001, with the total number of daily MMFS values $N=12428)$, is considered. It is found that $(a)$ the primary synodic period of the equatorial rotation of solar magnetic field, $P_{\odot}=26.929 \pm 0.015$ days, did not vary over the last 34 years, but $(b)$ the average intensity $H_{0}$ of the photospheric large-scale fields, by modulus, decreased by about $4.5 \%$ (with a confidence level of about $80 \%$ ). The conclusion is made that the longer, 90-year, cycle might be responsible for this potential gradual decrease of $H_{0}$. The average curve of MMFS variation as plotted with the primary rotational period $P_{\odot}$ demonstrates an obvious $N-S$ asymmetry of polarities, perhaps associated with the quadrupole component and "magnetic disequilibrium" of the Sun as a whole.
\end{abstract}

Key words. Sun: magnetic fields - Sun: rotation

\section{Introduction}

The mean magnetic field of the Sun (MMFS) represents the magnetic disequilibrium of the whole magnetic flux seen in the visible solar hemisphere. It is observed as a rule using the integrated solar flux without forming solar images at the spectrograph entrance slit. A magnetograph of high sensitivity is needed for the detection of small variations, from day to day, of the total (longitudinal) magnetic flux of the Sun.

During the last 34 years, more or less regular measurements of the Sun as a magnetic star were carried out at four observatories:

1) the Crimean Astrophysical Observatory (CrAO; program was started in 1968 by Severny 1969, with colleagues),

2) the Mount Wilson Observatory (MWO; observations were initiated by Scherrer 1973 and Howard 1974 in 1970),

3) the Wilcox Solar Observatory of the Stanford University (WSO; measurements were started in 1975 by J. Wilcox and P. Scherrer; see Scherrer et al. 1977b),

4) the Sayan Observatory of the Solar-Terrestrial Physics Institute (SO, Irkutsk; measurements were begun in 1982 by Grigoryev \& Demidov 1987).

We now have more than three decades of MMFS measurements - after the start of the Crimean program (Severny 1969; Kotov \& Severny 1983). These data, as shown below, can give us valuable information about the sector structure of the interplanetary magnetic field, "magnetic variability" of the Sun as a star and solar rotation, seen in the average magnetic field.

Send offprint requests to: V. I. Haneychuk,

e-mail: han@crao.crimea.ua
Recently Kotov et al. (1999) and Haneychuk (1999) showed that the power spectrum of MMFS variations is dominated by the period $P_{\odot}=26.92 \pm 0.02$ days, which demonstrates high stability of its initial phase over decades. This period characterises the rotation rate of the near-equatorial magnetic regions of the Sun and may be connected to the socalled "active longitudes". (Kotov \& Tsap 1999 also noticed to a close resonance, $7: 2$, between 26.92-day rotation of MMFS and orbital motion of Mercury, the closest planet to the Sun. It was suggested that this resonance have been formed at early stages of the formation of the solar system.)

In 1998-2001 we performed 396 daily measurements of MMFS, listed in Table 1. Combined with the previous $\mathrm{CrAO}$ data and those obtained in other three observatories, these measurements are analysed here to study the behaviour of the Sun during the new cycle 23 and over the total 34-year interval.

\section{Observations of MMFS, 1968-2001}

In each of four observatories the MMFS was registered by the solar magnetograph using the Zeeman effect of the photospheric absorption line Fe I $\lambda 525.02 \mathrm{~nm}$.

The magnetograph signal represents the mean strength of the longitudinal magnetic field $B$, measured in total light flux from the visible hemisphere of the Sun. The limb darkening enters $B$ as a weighting function, together with additional coefficients responsible for rotation, red shift of the spectral line near the limb, correlation of field strength with the line contour variations and photosphere brightness, etc. (see details in 
Table 1. The CrAO measurements of MMFS in 1998-2001 ( $B$ in Gs).

\begin{tabular}{|c|c|c|c|c|c|c|c|c|c|c|c|c|c|c|c|}
\hline Date & $B$ & Date & $B$ & Date & $B$ & Date & $B$ & Date & $B$ & Date & $B$ & Date & $B$ & Date & $B$ \\
\hline \multicolumn{2}{|c|}{ May 1998} & \multicolumn{2}{|c|}{ April 1999} & \multicolumn{2}{|c|}{ July 1999} & \multicolumn{2}{|c|}{ March 2000} & \multicolumn{2}{|c|}{ July 2000} & \multicolumn{2}{|c|}{ March 2001} & \multicolumn{2}{|c|}{ July 2001} & \multicolumn{2}{|c|}{ Oct. 2001} \\
\hline 26 & 0.21 & 17 & 0.52 & 28 & -0.55 & 31 & -1.54 & 21 & -0.62 & 27 & -0.66 & 6 & 0.26 & 11 & -0.63 \\
\hline \multicolumn{2}{|c|}{ June 1998} & 18 & 0.68 & 29 & -0.18 & April & 000 & 22 & -0.78 & 28 & -0.11 & 7 & 0.55 & 15 & -0.35 \\
\hline 2 & -0.70 & 20 & 0.29 & 30 & -0.37 & 2 & -0.99 & 24 & -1.18 & 30 & 0.23 & 8 & 0.43 & 18 & -0.09 \\
\hline 5 & -0.73 & 25 & -0.69 & Augus & 1999 & 3 & -0.68 & 25 & -0.60 & 31 & 0.17 & 9 & 0.06 & 19 & 0.31 \\
\hline 8 & 0.79 & 26 & -0.66 & 3 & -0.12 & 5 & 0.04 & 26 & -0.33 & \multicolumn{2}{|c|}{ April 2001} & 10 & -0.03 & 25 & -0.03 \\
\hline 16 & -0.74 & 30 & -0.55 & 4 & -0.19 & 6 & 0.43 & 28 & 0.31 & 3 & -0.16 & 11 & -0.32 & 26 & -0.33 \\
\hline 17 & -0.44 & \multicolumn{2}{|c|}{ May 1999} & 5 & -0.31 & 11 & 0.69 & 29 & 1.09 & 4 & -0.98 & 12 & 0.15 & 27 & -0.77 \\
\hline 18 & -0.31 & 1 & 0.39 & 6 & -0.17 & 12 & 0.29 & 30 & 1.43 & 7 & -0.91 & 13 & 0.70 & 28 & -0.55 \\
\hline 19 & -0.23 & 2 & 0.42 & 7 & -0.16 & 13 & 0.03 & 31 & 0.95 & 8 & -0.70 & 14 & 1.36 & 29 & -0.71 \\
\hline 23 & 0.33 & 4 & 0.75 & 8 & -0.16 & 14 & -0.02 & Sept. & 000 & 9 & -0.39 & 15 & 1.51 & Nov. & 001 \\
\hline 27 & -0.02 & 21 & -0.46 & 9 & 0.13 & 15 & 0.02 & 10 & 0.23 & 10 & -0.40 & 16 & 0.91 & 1 & 0.04 \\
\hline 28 & -0.71 & 22 & -0.50 & 10 & 0.11 & 17 & 1.14 & 12 & -0.31 & 11 & -0.63 & 17 & 0.90 & 2 & 0.04 \\
\hline 30 & -0.80 & 23 & -0.72 & 11 & 0.07 & 18 & 1.42 & 13 & -0.31 & 12 & -0.26 & 18 & 0.29 & 3 & -1.04 \\
\hline July & & 24 & -0.79 & 12 & 0.43 & 19 & 1.36 & 14 & -1.19 & 13 & -0.26 & 19 & 0.11 & 4 & -1.16 \\
\hline 1 & -0.61 & 28 & -0.19 & Sept. 1 & 999 & 21 & 0.72 & 15 & -1.51 & 20 & 0.09 & 20 & -0.57 & 5 & -0.59 \\
\hline 8 & 0.23 & 29 & -0.17 & 15 & 1.66 & 24 & -0.39 & 16 & -1.87 & 23 & 0.76 & 21 & -0.45 & 8 & 0.22 \\
\hline 12 & -0.39 & June 1 & & 17 & 0.30 & 26 & -0.69 & 17 & -1.65 & 27 & 0.29 & 22 & -0.31 & 9 & 0.33 \\
\hline 13 & -0.14 & 1 & 0.46 & 18 & -0.34 & 27 & -0.51 & 18 & -0.66 & 28 & -0.07 & 23 & -0.32 & Dec. & 01 \\
\hline 14 & -0.52 & 6 & -0.76 & 19 & -0.73 & 28 & -0.38 & 19 & 0.03 & 30 & -0.46 & 24 & -0.32 & 3 & -0.77 \\
\hline 15 & -0.28 & 7 & -0.54 & 20 & -1.15 & 29 & -0.48 & 21 & 1.34 & May & & Augu & 2001 & 5 & -0.34 \\
\hline 16 & -0.12 & 11 & 0.30 & 21 & -1.02 & 30 & -0.56 & 22 & 0.79 & 1 & -0.70 & 21 & 0.08 & 7 & -0.09 \\
\hline 19 & -0.23 & 14 & -0.21 & 22 & -0.39 & May & 00 & 28 & 1.02 & 2 & -1.06 & 23 & -0.01 & & \\
\hline 20 & -0.19 & 15 & -0.17 & 24 & 1.36 & 1 & -0.25 & 29 & 1.10 & 3 & -0.94 & 24 & -0.77 & & \\
\hline 21 & 0.17 & 16 & -0.34 & 25 & 1.20 & 3 & -0.13 & Oct. 2 & & 4 & -0.86 & 25 & -0.66 & & \\
\hline 22 & 0.11 & 17 & -0.17 & 27 & 0.80 & 4 & -0.10 & 2 & 0.03 & 6 & -0.74 & 26 & -0.60 & & \\
\hline 23 & 0.32 & 18 & -0.10 & 28 & 0.00 & June & 000 & 3 & 0.26 & 7 & -0.52 & 27 & 0.07 & & \\
\hline 31 & -0.10 & 19 & -0.07 & 29 & -0.65 & 7 & 0.11 & 4 & 0.19 & 8 & -0.34 & 28 & 0.22 & & \\
\hline Sept. & 998 & 21 & -0.21 & 30 & -0.85 & 8 & -0.31 & 5 & 0.24 & 10 & -0.14 & 29 & 0.34 & & \\
\hline 4 & -0.12 & 22 & 0.10 & Oct. 19 & & 9 & 0.01 & 6 & 0.64 & 15 & -0.04 & 30 & 0.23 & & \\
\hline 8 & -0.02 & 25 & 0.12 & 1 & -1.51 & 10 & 0.17 & 8 & 0.93 & 16 & -0.07 & 31 & -0.35 & & \\
\hline 11 & -0.25 & 26 & 0.51 & 2 & -1.40 & 11 & 0.40 & 13 & -1.83 & 17 & -0.30 & Sept. & 001 & & \\
\hline 13 & -0.17 & 28 & 0.30 & 3 & -0.52 & 12 & 0.47 & 14 & -2.24 & 18 & 0.28 & 1 & -0.46 & & \\
\hline 15 & 0.02 & 29 & 0.13 & 4 & -0.27 & 13 & 0.26 & 15 & -1.23 & 19 & 0.58 & 2 & -0.60 & & \\
\hline 18 & 0.17 & 30 & -0.01 & 5 & -0.28 & 14 & 0.71 & 16 & -0.40 & 21 & 0.67 & 5 & 0.28 & & \\
\hline 25 & 0.07 & July 19 & & 7 & 0.33 & 26 & -1.03 & 17 & 0.42 & 24 & 0.53 & 6 & 0.60 & & \\
\hline 27 & -0.93 & 1 & -0.59 & 12 & 1.71 & 27 & -0.49 & 24 & -0.43 & 25 & 0.33 & 10 & 0.45 & & \\
\hline 28 & -0.99 & 2 & -0.56 & 14 & 0.14 & 29 & 0.51 & 25 & 0.27 & 26 & 0.38 & 11 & 0.46 & & \\
\hline 29 & -0.55 & 4 & 0.04 & 18 & -1.10 & 30 & 0.36 & 26 & 0.01 & 27 & -0.21 & 13 & -0.30 & & \\
\hline Oct. & & 5 & -0.19 & 19 & -1.01 & July 2 & & 30 & -0.10 & 28 & -0.45 & 14 & -0.27 & & \\
\hline 2 & -0.20 & 6 & -0.38 & 23 & 0.96 & 1 & 0.97 & 31 & 0.38 & June & & 15 & 0.14 & & \\
\hline 6 & 0.38 & 7 & -0.25 & 24 & 0.61 & 2 & 1.04 & Nov. 2 & 00 & 3 & -0.42 & 16 & 0.19 & & \\
\hline 8 & -0.09 & 8 & -0.03 & 25 & 0.27 & 3 & 1.57 & 1 & 0.69 & 4 & -0.38 & 17 & -0.39 & & \\
\hline 9 & -0.22 & 9 & -0.26 & 29 & -0.89 & 4 & 0.85 & 2 & 0.64 & 8 & -0.67 & 18 & -0.40 & & \\
\hline 10 & -0.33 & 10 & 0.19 & Nov. 1 & 999 & 5 & 0.55 & 4 & 0.66 & 9 & 0.05 & 19 & -0.54 & & \\
\hline 12 & -0.46 & 11 & -0.29 & 3 & 0.46 & 6 & 0.45 & 5 & 0.09 & 10 & -0.05 & 20 & -0.64 & & \\
\hline 17 & 0.35 & 12 & -0.18 & 4 & 0.69 & 7 & 0.22 & 8 & -1.60 & 11 & 0.03 & 21 & -0.25 & & \\
\hline 18 & 0.84 & 13 & 0.04 & 5 & 1.14 & 8 & 0.81 & 9 & -1.76 & 12 & -0.02 & 22 & -0.06 & & \\
\hline 20 & 0.69 & 14 & -0.18 & 6 & 1.26 & 9 & 0.54 & 10 & -1.60 & 13 & -0.28 & 23 & 0.25 & & \\
\hline 22 & 0.14 & 15 & -0.06 & 7 & 1.45 & 10 & 0.64 & 11 & -1.18 & 14 & 0.14 & 24 & 0.55 & & \\
\hline 23 & -0.18 & 16 & -0.13 & 9 & 1.61 & 11 & 0.25 & 13 & 0.46 & 15 & 0.30 & 27 & 0.93 & & \\
\hline 24 & -0.52 & 20 & 0.83 & 10 & 1.32 & 12 & -0.13 & 14 & 1.09 & 18 & 0.67 & Oct. 2 & & & \\
\hline Nov. & 98 & 21 & 0.94 & 11 & 0.64 & 13 & -0.43 & 15 & 1.26 & 19 & 0.80 & 2 & -0.09 & & \\
\hline 3 & 0.24 & 22 & 1.07 & 12 & -1.88 & 14 & -0.26 & 16 & 1.05 & 20 & 0.30 & 3 & 0.27 & & \\
\hline 4 & -0.36 & 23 & 1.01 & 18 & 0.37 & 15 & -0.25 & 17 & 0.70 & 23 & -0.76 & 4 & 0.24 & & \\
\hline April & 999 & 24 & 0.70 & 22 & 0.30 & 16 & -0.64 & 18 & 0.18 & 29 & -0.85 & 5 & 0.12 & & \\
\hline 10 & 0.67 & 25 & 0.45 & March & 2000 & 17 & -0.93 & 20 & -0.22 & 30 & -1.35 & 8 & -0.60 & & \\
\hline 15 & 0.57 & 26 & -0.23 & 29 & -0.95 & 18 & -1.06 & 30 & 0.86 & July 2 & & 9 & -0.41 & & \\
\hline 16 & 0.51 & 27 & -0.45 & 30 & -1.28 & 19 & -1.04 & & & 5 & 0.40 & 10 & -0.58 & & \\
\hline
\end{tabular}


Table 2. Summary of MMFS data of four observatories.

\begin{tabular}{lcrccl}
\hline \hline Observatory & Interval, yrs & $N$ & $S$, Gs & $k$ & References \\
\hline CrAO (Crimea) & $1968-2001$ & 1945 & 0.665 & 0.948 & Kotov \& Severny (1983), Kotov et al. (1999, 1998a), Kotov \& \\
MWO (Mount Wilson) & $1970-1982$ & 2457 & 0.670 & 0.940 & $\begin{array}{l}\text { Kotov et al. (1998b) } \\
\text { WSO* (Stanford) }\end{array}$ \\
SO (Irkutsk) & $1975-2001$ & 7713 & 0.412 & 1.531 & $\begin{array}{l}\text { Solar-Geophysical Data Prompt Report 1977-2001 } \\
\text { Grigoryev et al. (1983), Grigoryev \& Demidov (1987), Kotov } \\
\text { et al. (1998a) }\end{array}$ \\
\hline Total & $1982-1993$ & 313 & 0.775 & 0.814 & \\
\hline
\end{tabular}

* WSO data for the interval May 16, 1975 - March 31, 2001.

** The total normalized dataset (see text).

Scherrer 1973; Grigoryev et al. 1983; Grigoryev \& Demidov 1987; Kotov et al. 1998b).

One single measurement of MMFS gives the $B$ value obtained by one of the instruments during the current UT-day. This is a magnetic disequilibrium of the visible hemisphere of the Sun which is proportional to the dominance of the magnetic flux of a given polarity over the opposite one.

The standard deviation $S$ was calculated for data set of each observatory, and then, using four $S$ values, the mean value $<S>=0.635$ Gs was determined. Then the $B$ values of each observatory were multiplied by a factor $k=\langle S\rangle / S$ (see Table 2). No additional coefficients, including the factor caused by the so-called "saturation" effect of magnetograph signal in the finestructured magnetic elements (Howard \& Stenflo 1972), were introduced.

After merging the normalized datasets we obtained the combined time series of the MMFS, 1968-2001, with the total number of daily values $N=12428$ and standard deviation $S=0.63$ Gs. This time series, if there is no special indication, is analysed below. The negative values of $B$ corresponds to the southern $(S)$ polarity, errors correspond to a standard $\pm 1 \sigma$ deviation, and initial $(0)$ phase to the UT moment $00^{\mathrm{h}} 00^{\mathrm{m}}$ on 1 January, 1968.

\section{Comparison of the CrAO and the WSO measurements}

In the Fig. 1 the original MMFS measurements of the $\mathrm{CrAO}$ and the WSO, carried out from June 1 through August 15, 1999 , are plotted. We can see more or less satisfactory qualitative agreement between variations of the two observatories. In general, the $\mathrm{CrAO}$ variations are essentially larger than those of the WSO. The agreement improves if one introduces the coefficient $\alpha=0.62$, equal to the ratio of the standard deviations of the two time series (see Table 2). For several days, or during a few consecutive days, however, large differences in $B$ values, exceeding errors of measurements, will still exist; they cannot be corrected by the factor $\alpha$ (the typical errors are: $\pm 0.15 \mathrm{Gs}$ for $\mathrm{CrAO}$ and $\pm 0.05 \mathrm{Gs}$ for WSO; on the accuracy of measurements see Grigoryev \& Demidov 1987; Kotov et al. 1998a, 1999).

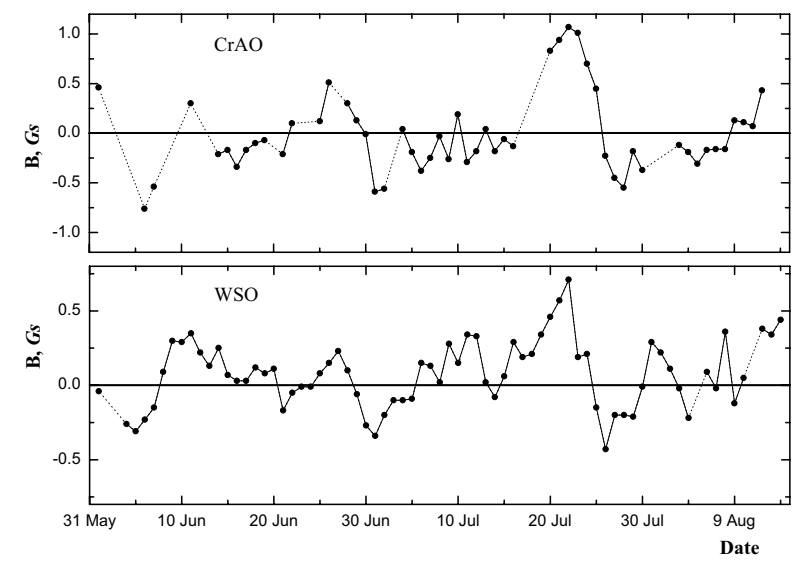

Fig. 1. Comparison of the MMFS data obtained from June 1 through August 15, 1999, at CrAO and WSO.

The equation of linear regression for $n=51$ pairs of the MMFS values obtained "simultaneously", i.e. on the same UT-day (Fig. 1), is:

$B(\mathrm{WSO})=0.06( \pm 0.03)+0.33( \pm 0.07) \times B(\mathrm{CrAO})$

( $B$ is in Gs, errors are indicated in parentheses). It follows that during the time span considered, the $\mathrm{CrAO}$ values, on average, were nearly three times larger than the WSO values. The correlation coefficient between these two sets is $r=0.59$. Earlier, Kotov et al. (1998a) reported that in 1991 the parameters of linear regression between the same pair of observatories revealed significantly different values:

$B(\mathrm{WSO})=0.30( \pm 0.07)+0.70( \pm 0.07) \times B(\mathrm{CrAO})$,

with the number of pairs of data $n=25$ and $r=0.94$.

The main causes of these discrepancies are displacement of the zero level of magnetographs, uncertainties in calibration, influence of instrumental polarization, inaccuracy of adjustment of optical elements and possible vignetting of the solar disk image.

Large scatter in MMFS measurements performed by different instruments might be also connected to the fact that we measure magnetic disequilibrium of the Sun. The main contribution to the MMFS signal is due to extended unipolar regions of background fields that have different weights. Those weights, together with instrumental effects, may substantially vary from day to day and from one instrument to another; these 
differences may also be caused by evolution of magnetic regions and solar rotation. Good approximation to real $B$ values can be attained, in our opinion, by merging data sets from different instruments - to get, as a result, the merged normalized MMFS series.

This question, however, needs special consideration. A wide-spreaded opinion that data obtained by some intruments are more accurate than those from other instrument (see Grigoryev \& Demidov 1987; Kotov et al. 1998a) seems to be questionable. Notice for instance that the $S$ values of three observatories - CrAO, MWO and SO (see Table 2) - are very close to each other and to their average value $0.70 \mathrm{Gs}$, whereas the Stanford measurements show $S=0.41 \mathrm{Gs}$, i.e. 1.7 times lower.

\section{Rotation of both MMFS and the Sun's equator}

During the last two decades, the interest in solar rotation has been related to the progress of helioseismology which collects much observational data and uses sophisticated methods to solve the problem of solar neutrino deficit and to study dynamics of convection and radiative zones of the Sun.

Sturrock \& Weber (2002), for example, found that solar neutrino flux varies with a synodic period $P_{v}=26.88$ days. The latest interpretation of such a variation may be that the neutrinos possibly can have simultaneous precession of both spin and flavor in the magnetic field (SPF-effect). For a given neutrino energy and density this effect may be enhanced due to the resonance process making "resonant spin flavor precession" (RSFP). Under these conditions the neutrino flux might be noticeably modulated by both inertial oscillations ( $r$-modes) in the radiation zone and rotation of the deep-seated magnetic field of the Sun.

Now we can directly determine the value of the main period of "rigid" rotation of the global magnetic field of the Sun using the MMFS data of 34 years. This period may reflect rotation of solar plasma near Sun's equator as well. Similar results based on a lesser amount of MMFS data were already obtained, e.g., by Kotov \& Tsap (1999), Kotov et al. (1999, 1998b), Haneychuk (1999).

The power spectrum calculated for the wide frequency range is shown in Fig. 2a, where major synodic periods, in days, are marked by numbers. Spectra are calculated both by direct Fourier transform and by the more complicated method of Scarge's (1982) periodogram that takes into account the irregularities in time series. Our data have a relatively small number of gaps in the time series, so the results of both spectra are practically identical. The left axis in Fig. 2 is power in $\mathrm{Gs}^{2}$, while the right axis is expressed in normalized power units $\eta=A_{\mathrm{h}}^{2} / \sigma^{2}$, where $\sigma$ is the standard deviation of the time series; see also Sturrock \& Weber (2002). The bottom axis is frequency in $\mu \mathrm{Hz}$ while the top one is expressed in cycles per year using the simple relationship

$v\left(\mathrm{yr}^{-1}\right)=31.55692 \cdot v(\mu \mathrm{Hz})$.

It is clearly seen that there are three groups of peaks, and the highest one corresponds to the Sun's rotation with a maximum value at 26.93 days. Two other groups correspond to second

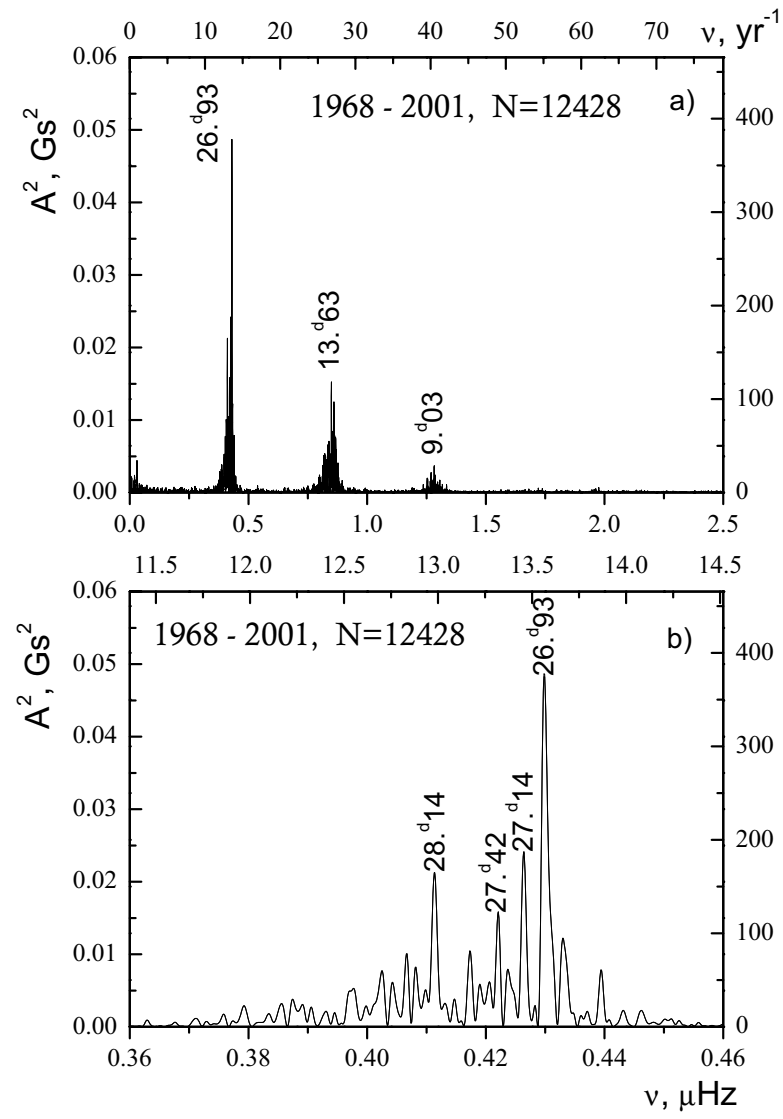

Fig. 2. Power spectrum of the 1968-2001 normalized MMFS data for a) the wide frequency range and b) domain near solar rotation frequencies. Power is represented by a square of harmonic amplitude, in $\mathrm{Gs}^{2}$ (left axis) and in normalized power units (right axis, see detail in Sturrock \& Weber 2002). The bottom axis is frequency in $\mu \mathrm{Hz}$ while the top one gives cycles per year. Main periods of rotation are indicated by numbers associated with the most prominent peaks.

and third overtones with the peak maxima near 13.63 days and 9.03 days, correspondingly (see details also in Haneychuk 1999). Some noticeable peaks are seen also in the lowfrequency region, e.g. that associated with a nearly 1 year period. These periodicities were discussed earlier by Kotov \& Levitsky (1983), Haneychuk (1995), Kotov et al. (1998a,b, 2002). Note that there are no substantial peaks within the highfrequency region up to the Nyquist limit $\approx 5.8 \mu \mathrm{Hz}$.

The periodicities marked in Fig. 2 are seen by eye in the original time series; as a result, the significance of each of those peaks is extremely high due to the low level of noise in the power spectrum (mean level, $\langle I\rangle \approx 1.3 \times 10^{-4} \mathrm{Gs}^{2}$ ). Using Scargle's (1982) technique, we can formally estimate, for example, a probability to get by chance the peak with a period of 9.03 days as $p \approx 3.7 \times 10^{-9}$, which corresponds to the confidence level $\mathcal{P} \approx 5.78 \sigma$ of a normal distribution. Possible influence of the window function on the results is also negligible, as was shown previously by Kotov et al. (1998b).

The power spectrum of MMFS computed for the frequency range near the solar rotation frequency is shown in Fig. 2b, where the maximal peak corresponds to the synodic period

$P_{\odot}=26.929 \pm 0.015$ days. 
The corresponding sidereal period is $P_{\odot}^{\prime \prime}=25.080 \pm 0.015$ days. In Fig. 2 we marked with numbers also the prominent peaks connected with differential rotation: $\approx 27.144,27.420$ and 28.140 days. One can see that main periodicity of the mean magnetic field of the Sun $P_{\odot}$ coincides within the error limits with the potential "neutrino" period $P_{v}=26.88 \pm 0.05$ days, presumably discovered in the GALLEX-GNO experimental data, 1991-2000, by Sturrock \& Weber (2002). Using the results of helioseismology, the authors also located the region in convective zone of the Sun that rotates with the same rate, see Fig. 8 in Sturrock \& Weber (2002). This is the lower region of the convective zone near the equator and a region near the surface at latitude $\approx 15^{\circ}$. It should be noted here that this coincides with results of Antonucci et al. (1990) who analysed rotation of the photospheric magnetic field using solar magnetograms and found that main rotation period in the northern hemisphere of the Sun is 26.9 days and is located in a zone with its center near $15^{\circ}$.

Period $P_{\odot} \approx 26.93 \approx 27.0$ days is also known as the equatorial rotation of solar plasma, the solar corona, photospheric magnetic field and interplanetary magnetic field (Wilcox et al. 1970; Antonucci \& Svalgaard 1974; Kotov et al. 1998b, 1999). According to Doppler measurements of Scherrer et al. (1980) the solar equator rotates with a synodic period of $26.96 \pm$ 0.17 days which is in fair agreement with our value for MMFS. We may therefore conclude that the most precise value of this period is $26.929 \pm 0.015$ days: using the total 34-year MMFS data we reduced its error by more than 10 times. One must note also that the main period of the solar magnetic rotation appears to be stable over more than three solar cycles, in spite of changing solar activity and polar magnetic field reversals. This long-term phase stability of rotation is probably connected to the phenomenon of so-called "active longitudes" of the Sun. It is then worth mentioning the results of Benevolenskaya et al. (1999) who found that magnetic fluxes of the "old" and "new" cycles are non-randomly distributed relative to "active longitudes".

Gilman \& Fox (1999) obtained a solution of dynamo equations taking into account the instability of the coexistence of differential rotation and a toroidal field near the base of the convective zone. According to their simulations we must observe sudden phase shifts of magnetic structures in the longitudinal direction in one or both hemispheres during the solar cycle, or essential changes in rotation of magnetic structures. Hence, the phase stability - over a few solar cycles - of the major rotation period $P_{\odot}$ of the Sun cannot be explaned by this theoretical approach.

Evidences in favour of time changes of solar rotation reported sometimes in the literature are connected usually with the phase of the solar 11-year cycle. It is possible to determine the rotation rate of the Sun for the first and second portions of the MMFS series separately, i.e. for the time spans 1968-1984 and 1985-2001. The corresponding two power spectra are similar to that plotted in Fig. 2 and are not shown here. As a result, we found that the same rotation period, within the error limits, dominated both time intervals:

1968-1984, $N=7157: P=26.94 \pm 0.03$ days, 1985-2001, $N=5271: P=26.97 \pm 0.03$ days.

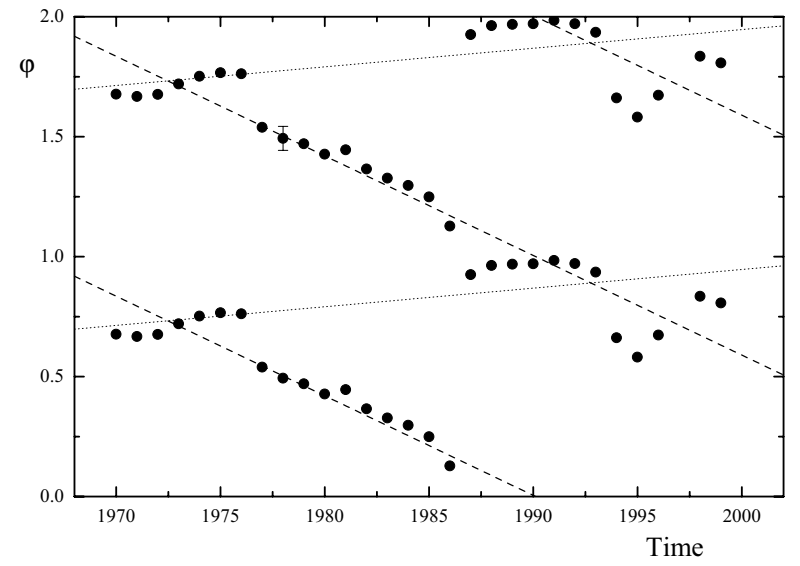

Fig. 3. Phase diagram conctructed with the test period $P_{\odot}^{\prime}=$ 27.000 days. Phases $\varphi$ are shown by points in the interval $0-1$ and are repeated for interval 1-2; the vertical bar indicates the typical error of the phase. Sloping dashed lines with negative slope correspond to the real period of $P_{\odot}=26.92 \pm 0.02$ days, while dotted lines with small positive slope correspond to the period $27.02 \pm 0.02$ days.

One can conclude therefore that the equatorial rate of the MMFS rotation and, probably, that of the whole Sun did not change over three cycles of solar activity covered by MMFS observations.

\section{The phase diagram}

Another way to get the precise value of periodicity is the phase diagram method. For each successive 5-year time interval we calculated the average phase curves for the test period $P_{\odot}^{\prime}=$ 27.000 days which is close to the main period in Fig. 2. Then the best-fit sinusoids were calculated for each curve, and amplitudes $A$ and phases $\varphi$ of harmonic maxima were determined.

The distribution of phases $\varphi$ with time is plotted in the Fig. 3 by dots (the average curve for the 1995-1999 interval with the mean time near 1997 happened to be insignificant and the corresponding dot is absent on the plot). The straight dotted lines of linear regression, calculated by the least-square method, shows that the best value of the period in question is equal to $P_{\odot}=26.92 \pm 0.02$ days, which coincides within the error limits with the previous value (4), obtained from the power spectrum analysis. One can also note that the phase of $P_{\odot}$-rotation appears to be stable, on average, over the total 34-year interval in spite of some deviations caused mainly by the 11-year cycle.

The phase diagram indicates however the presence of yet another periodicity, close to 27 days, too. The small positive run of phases (dots) can be seen within the intervals 1970-1976 and 1987-1993. Just for these intervals (14 data points only) the best-fit period is 27.03 days while for all data the period value is 27.02 days (corresponding dotted lines are shown in Fig. 3). As we can see, this period is present on the phase diagram, but it is practically absent in the power spectrum of Fig. 2b. Its value is very close to the mean value between the two most prominent peaks, of 27.14 and 26.93 days. The frequency difference between those two periods corresponds to $9.5 \pm 0.8$ years, which is close to the length of the 11-year 
cycle (the average length of the last 3 cycles is approximately 10.1 years). Probably, we have modulation of the main rotation period by the 11-year solar cycle. To answer this question we must have more data and make more specific analyses.

\section{The main rotation period}

It is interesting to compare the solar rotation rate (4) obtained for MMFS 1968-2001 with the characteristic rate for previous years. For this we can take results obtained earlier for the interplanetary magnetic field (IMF) as an indicator of rotation of the MMFS. It is well-known (Scherrer 1973; Scherrer et al. 1977a) that the sector structure of IMF is formed mainly by large-scale magnetic fields and thus correlates well with the sector structure of MMFS. The power spectrum of polarity of the IMF, published by Kotov (1987), shows that in 1926-1983 the structure of IMF near the ecliptic plane rotated preferably with two main periods: 28.20 and 26.94 days (rotation of the IMF over five solar cycles has been analysed also by Svalgaard \& Wilcox 1975). The first period, 28.20 days, characterizes probably rotation of the photospheric field at middle heliolatitudes or near the base of the convective zone. The second period we may compare with our value of $P_{\odot}$ :

IMF rotation, 1926-1983: period $26.940 \pm 0.010$ days, SMMF rotation, 1968-2001: period $26.929 \pm 0.015$ days.

Hence we may conclude that rotation of the MMFS and, as a consequence of the magnetic field freezing, that of the whole Sun at the equator, within the error limits, did not vary over the 76 year time span. The average value of these two periods which reflects the rotation of the Sun at the equator is

$P_{\odot}=26.935 \pm 0.010$ days.

The corresponding sidereal period is $P_{\odot}^{\prime \prime \prime}=25.085 \pm$ 0.010 days. We must note that such long-term stability of the photospheric field is difficult to explain in terms of the present conceptions about the origin of solar cyclicity where reversals of predominant polarity must be observed each 11 -year inter$\mathrm{val}$, as well as disappearance of the "old" polarity pattern being replaced by fields of a "new" cycle.

\section{Mean phase curve of the MMFS}

The phase and general view of the MMFS average curve might be interesting for solution of the problem of "active longitudes" of the Sun, and also for comparison with future solar observations. It is plotted in Fig. 4, where it seems worthwhile to note its non-harmonic form and asymmetry between positive and negative halfwaves. The latter might be associated with (a) quadrupole component of global solar magnetic field which can be comparable sometimes, in magnitude, with dipole component and can introduce significant deviations from a pure harmonic curve, and (b) the magnetic asymmetry of the Sun as a whole (see Severny 1968; Wilcox 1972; Kotov \& Levitsky 1985; Grigoryev \& Demidov 1989).

Parameters of the plotted curve are following: $A=0.22 \pm 0.03 \mathrm{Gs}, \varphi=0.85 \pm 0.02$.

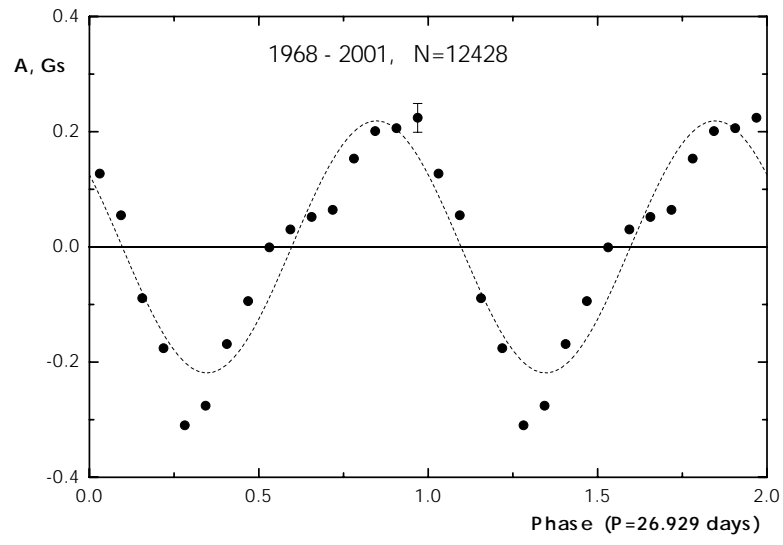

Fig. 4. The mean phase curve of the MMFS, 1968-2001, plotted with a period of $P_{\odot}=26.929$ days; the total number of MMFS measurements $N=12428$. The points represent the MMFS averages within phase bins of $P_{\odot} / 16$; the vertical error bar indicates the typical standard error, and the dotted curve represents the best-fit sinusoid.

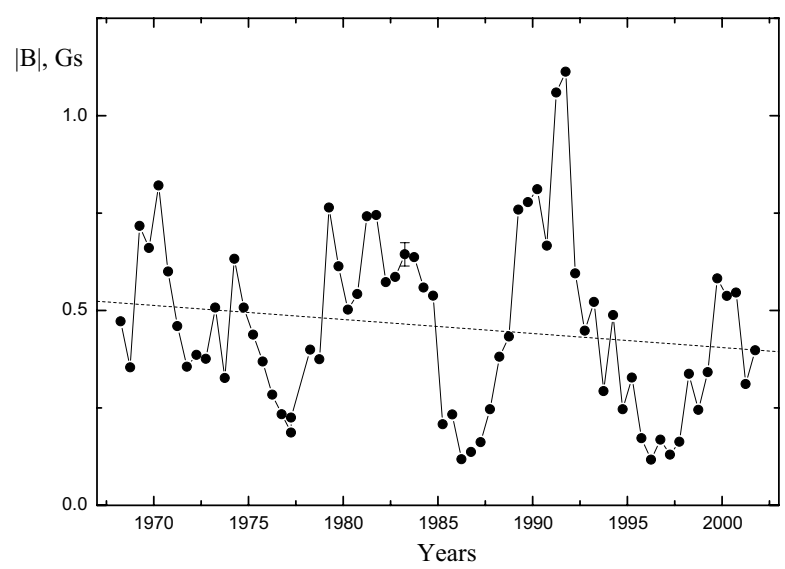

Fig. 5. Variations of the half-year averaged values of $|B|$ during last 34 years $(N=12428)$. Vertical error bar shows the typical error, dotted line is the linear trend, obtained by the least-square method.

\section{Slow time trend of the MMFS strength}

The MMFS is one of physical parameters which characterizes the solar cyclic activity equally with sunspot number. Is there some slow trend, up or down, of the MMFS absolute strength?

Lockwood et al. (1999) reported recently that the mean magnetic field of the solar corona increased almost by two times during the last 100 years. Kotov \& Kotova (2001) however, using direct measurements of Zeeman-effect of solar photosphere (i.e., the MMFS measurements) showed that any significant increase of the SMMF strength is absent. Using the full time series of the MMFS we can answer the question about long-term variation of the photospheric magnetic field of the Sun.

One should note that the study of various aspects of slow variations of the Sun as a star - both its radiation and magnetic field - appears to be important for solar-terrestrial relations, and also for the current problem of "global warming" of the Earth's climate (Parker 1999).

The normalized time series of the MMFS was converted to the series of absolute values $|B|(1968-2001, N=12428)$, with 
the resultant mean value $\langle|B|>=0.453$ Gs. We calculated then the running-mean values of $|B|$ for each half-year interval of the new time series. The behaviour of these half-year averages during last 34 years is shown in Fig. 5. Besides the main variation connected with the 11-year cycle, one can see the presence of an absolute maximum that occured in 1991.

The linear regression line

$|B|=0.513(0.028)-0.0036(0.0028) \times(t-1970.0)$

shows that there is a slow decrease of $|B|$ by $23 \pm 18 \%(|B|$ is in Gs, time $t$ is in years). The full amplitude of the decreasing effect is greater than one standard error, but its significance is quite low, with a confidence level of about $80 \%$. Since the MMFS measurements were made in four observatories, it seems unlikely that this slow trend of $|B|$ might be caused by some instrumental causes.

As we see from Fig. 5, the decrease of MMFS strength (and, consequently, of the average intensity of the Sun's global magnetic field) can be explained, in part, by a decrease of the MMFS strength in the cycle 23 , in spite of relatively large sunspot numbers in this new cycle.

\section{Conclusions}

The long-term measurements of the MMFS give the unique possibility to determine with high accuracy the rotation rate of the photospheric field and, therefore, of the atmosphere at/near solar equator. Using MMFS data for 34 years, also the data on polarity patterns of the IMF since 1926 (Svalgaard \& Wilcox 1975; Kotov 1987) we determined that the main sidereal period of Sun's rotation at the equator is

$P_{\odot}^{\prime \prime \prime}=25.085 \pm 0.010$ days;

this value did not vary, within the error limits, over last 76 years.

That fact that main rotation period of the magnetic field of the Sun coincides within error limits with the major variation of solar neutrino flux, detected by Sturrock \& Weber (2002), indicates the reality of solar neutrino deviations in the strong magnetic field inside the convective zone. This fact may also suggest that below the convective zone the magnetic field is relatively small.

The fact that dominating - in rotation - magnetic structure did not "dissipate", and the corresponding peak in the power spectrum is not split, arises the important question of the actual processes of generation and rotation of the global solar magnetic field which appears to be invariable with respect to the phase of the 11-year cycle. It seems hard to find a simple explanation of this phenomenon in terms of the present dynamo theory.

The average strength of the MMFS decreased by nearly $4.5 \%$ during the last 34 years. This decrease however has low (about $80 \%$ ) statistical significance, and must be verified by new measurements. If real, it might be caused by the longterm solar variabiliy cycle, with a quasi-period near or larger than 90 years.
Acknowledgements. We are very grateful to N. P. Rusak for the technical work at the CrAO telescope STT-1 and with the solar magnetograph, and also to N. G. Sunitza for the help in MMFS data reduction. We thank M. L. Demidov for exchange of the MMFS data and to J. Staude and J. O. Stenflo for the fruitful discussions of problems of the MMFS measurements and magnetic variability of the Sun. The authors also are very thankful to the referee Peter Sturrock for his helpful comments and suggestions which essentially improved the paper. Our special thanks to the anonymous staff of A\&A for numerous corrections in English. This work was supported in part by the INTAS grant No. 2000-840.

\section{References}

Antonucci, E., \& Svalgaard, L. 1974, Sol. Phys., 34, 3

Antonucci, E., Hoeksema, J. T., \& Scherrer, P. H. 1990, ApJ, 360, 296

Benevolenskaya, E. E., Hoeksema, J. T., Kosovichev, A. G., \& Scherrer, P. H. 1999, ApJ, 517, L163

Gilman, P. A., \& Fox, P. A. 1999, ApJ, 522, 1167

Grigoryev, V. M., \& Demidov, M. L. 1987, Sol. Phys., 114, 147

Grigoryev, V. M., \& Demidov, M. L. 1989, in Solar Magnetic Fields and Corona, 1 (Novosibirsk, Nauka), 108

Grigoryev, V. M., Demidov, M. L., \& Osak, B. F. 1983, Issled. po geomagnetizmu, aeronomii i fizike Solntsa, 65, 13 (in russian)

Haneychuk, V. I. 1995, Bull. Crimean Astrophys. Obs., 92, 84

Haneychuk, V. I. 1999, AZh, 76, 385

Howard, R. 1974, Sol. Phys., 38, 283

Howard, R., \& Stenflo, J. O. 1972, Sol. Phys., 22, 402

Kotov, V. A. 1987, Bull. Crimean Astrophys. Obs., 77, 39

Kotov, V. A., \& Kotova, I. V. 2001, Soviet Astron. Lett., 27, 302

Kotov, V. A., \& Levitsky, L. S. 1983, Bull. Crimean Astrophys. Obs., 66,110

Kotov, V. A., \& Levitsky, L. S. 1985, Bull. Crimean Astrophys. Obs., 71,32

Kotov, V. A., \& Severny, A. B. 1983, Mean Magnetic Field of the Sun as a star. Catalogue 1968-1976, Soviet Geophysical Committee, Moscow (in russian)

Kotov, V. A., \& Tsap, T. T. 1999, Kinematics and Physics of Cel. Bodies, 15, 1

Kotov, V. A., Demidov, M. L., Haneychuk, V. I., \& Tsap, T. T. 1998a, Bull. Crimean Astrophys. Obs., 94, 110

Kotov, V. A., Scherrer, P. H., Howard, R. F., \& Haneychuk, V. I. 1998b, ApJS, 116, 103

Kotov, V. A., Haneychuk, V. I., \& Tsap, T. T. 1999, AZh, 76, 218

Kotov, V. A., Haneychuk, V. I., \& Tsap, T. T. 2002, Kinematics and Physics of Cel. Bodies, 18, 205

Lockwood, M., Stamper, R., \& Wild, M. N. 1999, Nature, 399, 437

Parker, E. N. 1999, Nature, 399, 416

Scargle, J. D. 1982, ApJ, 263, 835

Scherrer, P. H. 1973, Stanford Univ. Inst. Plasma Res. Rep., 554

Scherrer, P. H., Wilcox, J. M., Kotov, V., Severny, A. B., \& Howard, R. 1977a, Sol. Phys., 52, 3

Scherrer, P. H., Wilcox, J. M., Svalgaard, L., et al. 1977b, Sol. Phys., 54,353

Scherrer, P. H., Wilcox, J. M., \& Svalgaard, L. 1980, ApJ, 241, 811

Severny, A. B. 1968, Bull. Crimean Astrophys. Obs., 38, 3

Severny, A. 1969, Nature, 224, 53

Sturrock, P. A., \& Weber, M. A. 2002, ApJ, 565, 1366

Svalgaard, L., \& Wilcox, J. M. 1975, Sol. Phys., 41, 461

Wilcox, J. M. 1972, Comm. Astrophys. Space Phys., 4, 141

Wilcox, J. M., Schatten, K. H., Tanenbaum, A. S., \& Howard, R. 1970, Sol. Phys., 14, 255 